\title{
Relevance of Colonic Gas Analysis and Transit Study in Patients With Chronic Constipation
}

\author{
Seon-Young Park, Hyun-Bum Park, Ji-Myung Lee, Ho-Jun Lee, Chang-Hwan Park, Hyun-Soo Kim, Sung-Kyu Choi, and \\ Jong-Sun Rew*
}

Department of Internal Medicine, Chonnam National University Medical School, Gwangiu, Korea

\section{Background/Aims}

Colon transit time (CTT) is a useful diagnostic tool in chronic constipation, but requires good patient compliance. We analyzed the correlation between the gas volume score (GVS) and CTT in patients with chronic constipation.

\section{Methods}

The study included 145 consecutive patients $(65 \mathrm{men})$ with chronic constipation. The primary outcome was the correlation between the colon GVS and CTT. Secondary outcomes were the differences in colon GVS according to CTT and subtypes of chronic constipation.

\section{Results}

There were 81 patients with "CTT $<45$ hours" and 64 patients with "CTT $\geq 45$ hours." In addition, 88 patients were classified as having functional constipation and 57 were classified as having constipation predominant irritable bowel syndrome (IBS-C). There was no significant correlation between CTT and colon GVS. However, the right colon GVS showed a positive correlation with right $\mathrm{CTT}(r=0.255, P=0.007)$. The median total colon GVS was significantly higher in patients with "CTT $\geq 45$ hours" than in those with "CTT $<45$ hours" $(5.65 \%$ vs $4.15 \%, P=0.010)$. There were no significant differences in colon GVS between the functional constipation and IBS-C.

\section{Conclusions}

We were unable to detect a correlation between GVS and CTT in patients with chronic constipation. However, total colon GVS may be a method of predicting slow transit in patients with chronic constipation.

\section{(J Neurogastroenterol Motil 2015;21:433-439)}

\section{Key Words}

Colon; Constipation; Gastrointestinal transit; Irritable bowel syndrome

Received: September 21, 2014 Revised: December 21, 2014 Accepted: December 30, 2014

(c) This is an Open Access article distributed under the terms of the Creative Commons Attribution Non-Commercial License (http://creativecommons. org/licenses/by-nc/4.0) which permits unrestricted non-commercial use, distribution, and reproduction in any medium, provided the original work is properly cited.

${ }^{*}$ Correspondence: Jong-Sun Rew, MD, PhD Department of Internal Medicine, Chonnam National University Medical School, 42 Jebongro, Dong-gu, Gwangju 501-757, Korea Tel: +82-62-220-6296, Fax: +82-62-225-8578, E-mail: jsrew@jnu.ac.kr

Seon-Young Park and Hyun-Bum Park contributed equally to this work.

Financial support: None.

Conflicts of interest: None.

Author contributions: Seon-Young Park, planning and/or conducting the study, and drafting the manuscript; Hyun-Bum Park, Ji-Myung Lee, Ho-Jun Lee, Chang-Hwan Park, Hyun-Soo Kim, and Sung-Kyu Choi; and Jong-Sun Rew, in planning the study, revision of the manuscript. 


\section{Introduction}

Assessment of colon transit is important in patients with symptoms of colonic dysmotility because it can provide useful mechanistic insights and gauge treatment response. ${ }^{1,2}$ Among various available tests, radiopaque marker tests have traditionally been performed to measure colon transit time (CTT), representing the most widely used method. Transit studies using radiopaque markers distinguish constipation subgroups such as normal or slow transit constipation and assess segmental transit times in patients with delayed total colon transit. ${ }^{3}$ However, this method requires good patient compliance. At times, elderly patients tend to forget to take the markers or undergo abdominal radiography as scheduled.

However, intestinal bowel gas can easily be identified on plain abdominal radiographs. Bowel gas volume has been reported to represent an important indicator of bowel function in bowel diseases including chronic constipation. Bowel gas was significantly greater in patients with functional bowel disorder than in controls. ${ }^{4-6}$ Patients with postoperative bowel dysfunction such as constipation displayed relatively high volumes of gas in the left colon. Left colon transit time correlated with left colon gas volume score after colorectal surgery. ${ }^{7}$ Furthermore, Pimental et al ${ }^{8}$ demonstrated that methane $\left(\mathrm{CH}_{4}\right)$ gas slows intestinal transit and alters intestinal motor function. $\mathrm{CH}_{4}$ excretion was associated with chronic constipation. ${ }^{9}$ If the bowel gas volume might be associated with colon transit, clinician could use bowel gas volume score (GVS) instead of somewhat complex CTT for evaluation of colon transit.

In this study, we investigated if the bowel gas volume score (GVS) might substitute for CTT. We therefore analyzed the correlation between the colon GVS and CTT in patients with chronic constipation.

\section{Patients and Methods}

\section{Patients}

Consecutive patients admitted to Chonnam National University Hospital for colon transit studies over an 8-year period (from Jan 2005 to Jan 2013) were included in the study, provided they had suffered from chronic constipation. Exclusion criteria were known organic intestinal disease, prior abdominal surgery, or pregnancy. The cohort included patients with constipation pre- dominant irritable bowel syndrome (IBS-C) and patients with functional constipation according to Rome III criteria.

\section{Assessment of Colon Transit Time}

CTT was assessed using radiopaque markers (Kolomark ${ }^{\mathrm{TM}}$, M.I Tech, Pyeongtaek-si, Korea). Each gelatin capsule contained 20 ring-shaped radiopaque markers measuring $1 \mathrm{~mm} \times$ $4.5 \mathrm{~mm}$. Each radiopaque polyethylene marker contained $40 \%$ barium.

Subjects ingested 1 capsule at 9 AM on days 1, 2, and 3. On days 4 and 7, plain abdominal radiographs were obtained in the erect position. Patients were asked to maintain their regular diets and to refrain from using laxatives during the study. The projection zones in the right colon, left colon, and rectosigmoid are limited by the bony landmarks (fifth lumbar vertebra, the pelvic outlet) as described by Arhan et al. ${ }^{10}$ Markers were readily identified and counted in each segment on abdominal radiographs. CTT in each segment and throughout the entire colon was calculated by multiplying the number of markers by 1.2. ${ }^{11} \mathrm{CTT}$ s were denoted as right colonic transit time (RCTT), left colonic transit time (LCTT), and rectosigmoid colonic transit time (RSCTT). Patients were classified into 2 groups (CTT $\leq 45$ hours vs CTT $>45$ hours according to their CTTs.

\section{Gas Volume Analysis of the Colon From Abdominal Radiographs}

Each film used for the transit study was obtained using a picture archiving and communication system (PACS; Maroview version 5.4, MAROTECH Inc, Seoul, Korea). Areas of colon gas were outlined as rectangular and by free-hand drawing a region of interest and calculated. The large intestine was divided into 3 parts, as mentioned above. According to the GVS defined by Koide, ${ }^{12}$ the percentage (\%) of right colon gas in the region surrounded by a horizontal line tangential to the suprasymphysary margin, a horizontal line tangential to the uppermost diaphragm, and the most lateral line tangential to the right and left costal arches was defined as the right colon GVS (RCS). The left colon (LCS) and rectosigmoid colon (RSCS) scores were defined in a similar manner. Kerckring folds were useful in distinguishing small bowel gas from colon gas when small bowel gas was present. No patients in this series were gasless.

\section{Outcome Measures}

The primary outcome was the correlation between the colon GVS and CTT in patients with chronic constipation. The secon- 
dary outcomes were as follows: (1) the difference in colon GVS according to colon transit and (2) the difference in colon GVS between functional constipation and IBS-C patients.

\section{Statistical Methods}

Statistical analysis was performed with IBM SPSS Statistics version 20.0 (IBM Co, Armonk, NY, USA). Categorical variables were described using frequencies and percentages. Continuous variables were summarized using median values (quartiles). Between-group comparisons were performed using the Mann-Whitney signed-rank test, the Wilcoxon signed-rank test, and the Chi-squared test, as appropriate. Relationships between GVS and CTT were assessed by the Pearson's product moment correlation coefficient test. To assess the ability of each GVS to predict colon transit, the area under the receiver operating characteristics (ROC) curve was tested. Statistical significance was established at the level of $P<0.05$.

\section{Results}

A total of 157 patients with chronic constipation underwent the colon transit study. Of these, 12 patients were excluded due to an operative history for gastrointestinal malignancy in 5 cases and incomplete colon transit studies in 7 cases. Ultimately, 145 patients (65 men, mean age $55.6 \pm 17.9$ years) were included. These included $88(60.7 \%)$ patients classified as having "functional constipation" and 57 (39.3\%) patients classified as having "IBS-C." There were no differences in age, gender, and total CTT between the "functional constipation" and "IBS-C" groups (Table 1). There were 81 (55.9\%) patients with "CTT<
45 hour" and 64 (44.1\%) patients with "CTT $\geq 45$ hours."

\section{Colon Transit Time and Colon Gas Volume Score}

There was no correlation between CTT and colon GVS. However, RCS showed a positive correlation with RCTT ( $r=$ $0.255, P=0.007)$. There were no correlations between LCTT and LCS and between RSCTT and RSCS $(P=0.168$ and 0.475 , respectively; Fig. 1).

\section{Colon Gas Volume Score According to Colon Transit}

The Median CTT was 22.8 hours (6.6-32.4 hours) in patients with "CTT $<45$ hours" and 73.8 hours (56.7-93.3 hours) in patients with "CTT $\geq 45$ hours." The median total colon GVS was significantly higher in patients with "CTT $\geq 45$ hours" than in those with "CTT $<45$ hours" ( $5.66 \%$ vs $4.15 \%$, $P=0.010$; Fig. 2). There was no difference in RCS between the 2 groups $(P>0.05)$. However, there were significant difference in LCS and RSCS (Table 2). The ROC curve analysis showed a fair result for the total colon GVS predicting slow transit in patients with chronic constipation. The ROC analysis, the optimal cut-off value to diagnosis slow CTT (over 45 hours) was $4.63 \%$ (sensitivity $66.7 \%$, specificity $54.5 \%$, and AUC 0.628 [95\% confidence interval, 0.535 and 0.721 ]; Fig. 3).

\section{Colon Gas Volume Score Between Functional Constipation and Constipation Dominant Irritable Bowel Syndrome}

There were no significant differences in colon GVS between

Table 1. Colon Transit Time and Colon Gas Volume Score of 145 Patients

\begin{tabular}{lccc}
\hline \multicolumn{1}{c}{ Variable } & $\begin{array}{c}\text { Functional constipation } \\
(\mathrm{n}=88)\end{array}$ & $\begin{array}{c}\text { IBS-C } \\
(\mathrm{n}=57)\end{array}$ & $\begin{array}{c}P \text {-value } \\
\text { Slow transit (n [\%]) }\end{array}$ \\
TCTT (median [quartile], hr) & $53(60.2)$ & $35(61.4)$ & 0.890 \\
RCT (median [quartile], hr) & $83.8(15.6-74.1)$ & $38.4(21.0-67.2)$ & 0.550 \\
LCTT (median [quartile], hr) & $8.4(1.2-17.7)$ & $9.6(1.8-17.4)$ & 0.961 \\
RSCTT (median [quartile], hr) & $18.6(3.6-38.1)$ & $8.4(2.4-14.4)$ & 0.903 \\
Colon GVS (median [quartile], \%) & $4.92(3.39-0.68)$ & $14.4(5.4-39.0)$ & 0.693 \\
RCS (median [quartile], \%) & $1.60(0.93-2.61)$ & $5.03(2.50-7.05)$ & 0.125 \\
LCS (median [quartile], \%) & $1.95(1.12-2.92)$ & $1.73(0.88-3.50)$ & 0.294 \\
RSCS (median [quartile], \%) & $0.65(0.02-1.55)$ & $1.12(0.56-3.37)$ & 0.951 \\
\hline
\end{tabular}

IBS-C, constipation dominant irritable bowel syndrome; TCTT, total colon transit time; RCTT, right colon transit time; LCTT, left colon transit time; RSCTT, rectosigmoid colon transit time GVS, gas volume score; RCS, right colon gas volume score; LCS, left colon gas volume score; RSCS, rectosigmoid colon gas volume score. 

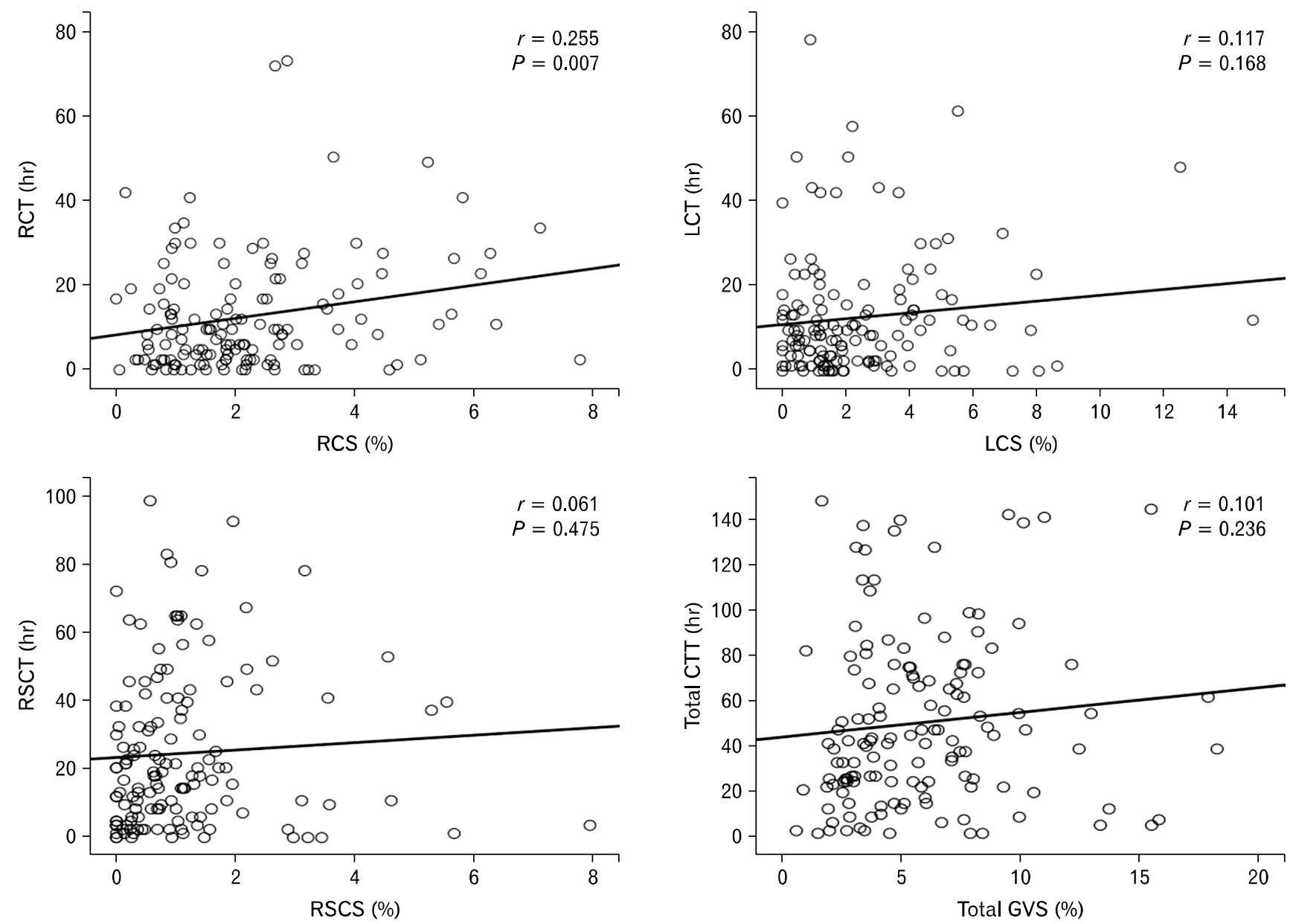

Figure 1. Relationship between colon transit time (CTT) and colon gas volume score (GVS). There was no correlation between CTT and total colon GVS. However, right colon gas volume score (RCS) showed a positive correlation with right colon transit time $(\mathrm{RCTT} ; r=0.255, P=0.007)$. LCTT, left colon transit time; LCS, left colon gas volume score; RSCTT, rectosigmoid colon transit time; RSCS, rectosigmoid colon gas volume score.

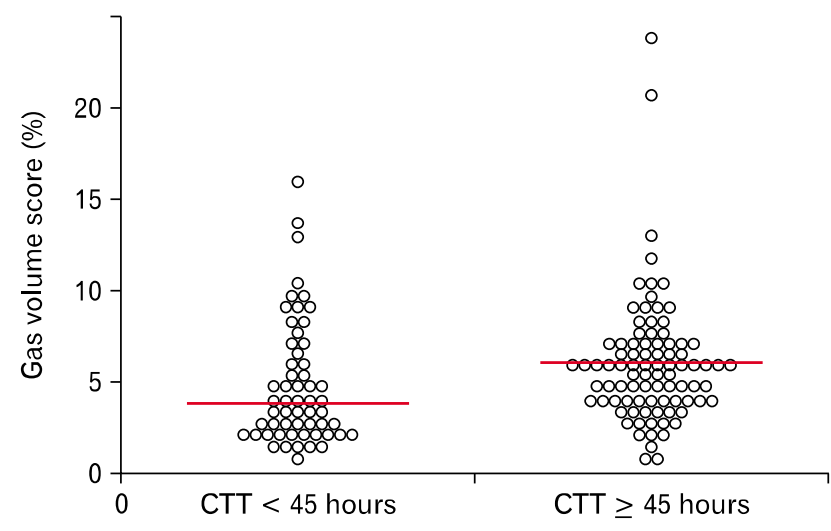

Figure 2. Colon gas volume score (GVS) according to colon transit. The median total colon GVS was higher in patients with "CTT $\geq 45$ hours" than in those with "CTT $<45$ hours" ( $5.66 \%$ vs $4.15 \%, P=$ $0.010)$. patients with functional constipation and those with IBS-C (Table 1).

\section{Discussion}

In the present study, total colon GVS did not correlate with CTT, but we found a positive, although low, correlation between RCTT and RCS. In the subgroup analysis of patients with slow transit, only RCS correlated with RCTT. Intestinal gases produced by enteric microflora are associated with various abdominal symptoms such as bloating, abdominal pain, and bowel habit changes. Intestinal gases produced by enteric bacteria include $\mathrm{CH}_{4}$, hydrogen $\left(\mathrm{H}_{2}\right)$, hydrogen sulfide $\left(\mathrm{H}_{2} \mathrm{~S}\right)$, and carbon dioxide $\left(\mathrm{CO}_{2}\right) .{ }^{13}$ Among these gases, $\mathrm{CH}_{4}$ gas slows intestinal transits, augments reflex contraction of the intestinal muscle, and al- 
Table 2. Gas Volume Score According to Colon Transit

\begin{tabular}{lccc}
\hline \multicolumn{1}{c}{ Variable } & $\begin{array}{c}\text { CTT }<45 \text { hours } \\
(\mathrm{n}=81)\end{array}$ & $\begin{array}{c}\text { CTT } \geq 45 \text { hours } \\
(\mathrm{n}=64)\end{array}$ & $\begin{array}{c}P \text {-value } \\
\text { Total GVS (median [quartile], \%) }\end{array}$ \\
RCS (median [quartile], \%) & $4.15(2.63-6.68)$ & $5.66(3.90-7.09)$ & 0.010 \\
LCS (median [quartile], \%) & $1.60(0.89-3.09)$ & $1.79(1.00-3.16)$ & 0.555 \\
RSCS (median [quartile], \%) & $1.44(0.81-2.43)$ & $2.13(0.95-3.80)$ & 0.023 \\
\end{tabular}

CTT, colon transit time; GVS, gas volume score; RCS, right colon gas volume score; LCS, left colon gas volume score; RSCS, rectosigmoid gas volume score.

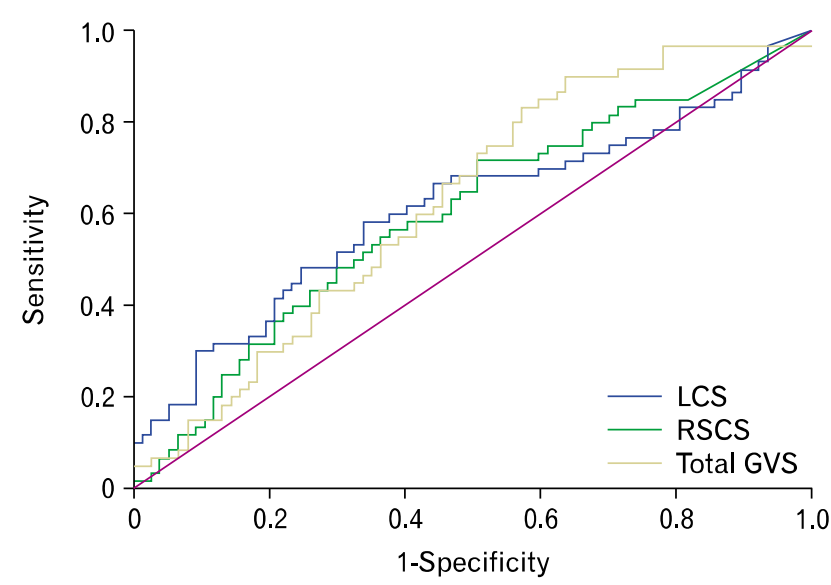

Figure 3. Receiver operating characteristics (ROC) curve for predicting slow transit constipation. The ROC of the model (total colon gas volume score [GVS]) is shown, with an area under the ROC curve of 0.628 (95\% confidence interval, 0.535 and 0.721 ). LCS, left colon gas volume score; RSCS, rectosigmoid gas volume score.

ters intestinal motor function. ${ }^{8}$ In addition, Jahng et $\mathrm{al}^{14}$ showed that $\mathrm{CH}_{4}$ gas delayed the contraction velocity of ileal peristalsis and that $\mathrm{H}_{2}$ gas shortened colonic transit in the proximal and distal colon. $\mathrm{CH}_{4}$ positivity was associated with delayed colonic transit or prolonged oro-cecal transit time. ${ }^{15-17}$ Moreover, $\mathrm{CH}_{4}$ producers had significantly higher GVS as compared with nonproducers. ${ }^{18}$ In this study, total colon gas volume did not correlate with CTT. And, only RCS correlated with RCTT. The reasons would be as follows: colon contains not only $\mathrm{CH}_{4}$ but also $\mathrm{H}_{2}$. The component of variable intestinal gases may affect (delay or shorten) colon transit. Moreover, GVS measurement in the left colon and rectosigmoid colon may be more influenced by gas from the stomach or small intestine than measurement in the right colon. The retroperitoneal structure of the colon (ascending colon, descending colon, and rectum) are relatively constant in position. These are often more readily identified than transverse colon or sigmoid colon which are more variable in position. Even though we try to differentiate small bowel gas from colonic gas, redundant transverse colon or sigmoid colon frequently overlaps with small bowel. For this reason, only RCS might positively correlate with right CTT. Another possible hypothesis is that methanogenic bacteria may be abundant in the right colon. However, previous study showed that a few methanogenic bacteria were dominantly found in the left colon. ${ }^{19,20}$ Therefore, the analysis for component of gases and gas producing bacteria needs to be studied.

We divided patients with chronic constipation into 2 groups (CTT $<45$ hours vs CTT $\geq 45$ hours) according to CTT (45 hours). CTTs are generally shorter in the normal Asian population than in Western populations. In most Western studies, the mean CTT was 30-40 hours with upper normal limit of 70 hours. ${ }^{411,21}$ In studies from Asia, the mean CTT was 20-30 hours in normal subjects. ${ }^{2,22,23}$ Among these studies, Chan et al ${ }^{22}$ suggested that upper limit of normal CTT (mean value $+2 \mathrm{SD}$ ) was 62 hours. In this study, we assumed that upper limit of normal CTT was 45 hours (mean value $+1 \mathrm{SD}$ ). We therefore discretionally categorized patients into "CTT $<45$ hours" and "CTT $\geq 45$ hours."

We showed that total colon GVS was higher in patients with "CTT $\geq 45$ hours" than in those with "CTT $<45$ hours" $(5.66 \%$ vs $4.15 \%)$. Bowel gas is easily identified on plain abdominal radiographs, and is useful for diagnosing ileus and other diseases. ${ }^{6}$ Several previous studies showed that bowel gas volume represented an important indicator of bowel function. ${ }^{4,12,24}$ However, until now, there have been few reports on the associations between colon GVS and colon transit. Although ROC curve could not show the diagnostic value of colon GVS in predicting slow transit and the range of colon GVS was largely overlapping each other, this study may suggest the role of colon GVS as supportive tool for diagnosis of slow transit constipation. When clinicians encounter a constipated patient with a large amount of bowel gas on plain abdominal radiography, they can suspect that the patient may have slow colon transit.

In this study, mean colon transit time was 43.8 hours in pa- 
tients with functional constipation and 38.4 hours in patients with IBS-C. And, $60.6 \%$ patients were categorized as "slow transit" (CTT $>45$ hours). A study on Iranians with constipation, $57.8 \%$ had slow total CTT and mean total CTT in patients with functional constipation and IBS-C was 52.2 hours and 41.2 hours, respectively. ${ }^{25}$ Mollen et $\mathrm{al}^{26}$ showed that $71 \%$ of patients with functional constipation had abnormal total CTT, however, patients with IBS had normal colon transit. Actually, previous study showed that only $12 \%$ of IBS-C had delayed colon transit. However, in that study, stool form and stool frequency were negatively correlated to total CTT. ${ }^{27}$

We showed that CTT was not different between patients with functional constipation and those with IBS-C. A few studies compared the CTT of functionally constipated and IBS-C patients, and showed conflicting data. Two studies showed that the RSCTT was significantly higher in patients with functional constipation than in those with IBS-C. ${ }^{25,28}$ In our study, patients with functional constipation had a higher RSCTT (18.6 hours) than patients with IBS-C (14.4 hours). However, there was no significant difference between the 2 groups.

This study has some limitations as follows: (1) this was a retrospective study with a relatively small sample size. Therefore, we could not show subjects' individual symptoms or symptom scores. (2) Bowel gas area on plain abdomen radiographs does not constitute an accurate real gas volume. And, we adopted a variant method of the original GVS. ${ }^{12}$ Even though we try to differentiate small bowel gas from colonic gas, redundant transverse colon or sigmoid colon frequently overlaps with small bowel. (3) There was the only small mean difference of colon GVS between the 2 groups. And, ROC curve could not show the diagnostic value of colon GVS in predicting slow transit. Therefore, at the present time, it is difficult to apply to clinical situation on managing constipated patient. For clinical impact on managing constipated patients, the method for measurement of colon GVS need to be more validated and computerized.

In summary, there was no correlation between CTT and colon GVS. However, colon GVS in patients with "CTT $\geq 45$ hours" was higher than that in patients with "CTT $<45$ hours."

In conclusion, we were unable to detect a correlation between the colon GVS and CTT in patients with chronic constipation. However, total colon GVS may be a method of predicting slow transit in patients with chronic constipation.

\section{References}

1. Velde SV, Notebaert A, Meersschaut V, Herregods N, Van Winckel M, Van Biervliet S. Colon transit time in healthy children and adolescents. Int J Colorectal Dis 2013;28:1721-1724.

2. Kim ER, Rhee PL. How to interpret a functional or motility test colon transit study. J Neurogastroenterol Motil 2012;18:94-99.

3. Rao SS, Camilleri M, Hasler WL, et al. Evaluation of gastrointestinal transit in clinical practice: position paper of the American and European Neurogastroenterology and Motility Societies. Neurogastroenterol Motil 2011;23:8-23.

4. Chami TN, Schuster MM, Bohlman ME, Pulliam TJ, Kamal N, Whitehead WE. A simple radiologic method to estimate the quantity of bowel gas. Am J Gastroenterol 1991;86:599-602.

5. Leech SC, McHugh K, Sullivan PB. Evaluation of a method of assessing faecal loading on plain abdominal radiographs in children. Pediatr Radiol 1999;29:255-258.

6. Starreveld JS, Pols MA, Van Wijk HJ, Bogaard JW, Poen H, Smout AJ. The plain abdominal radiograph in the assessment of constipation. Z Gastroenterol 1990;28:335-338.

7. Seike K, Koda K, Oda K, Kondo E, Ishizuka M, Miyazaki M. Relevance of abdominal gas analysis and transit study after colorectal cancer surgery. Scand J Gastroenterol 2003;38:387-391.

8. Pimentel M, Lin HC, Enayati P, et al. Methane, a gas produced by enteric bacteria, slows intestinal transit and augments small intestinal contractile activity. Am J Physiol Gastrointest Liver Physiol 2006; 290:G1089-G1095.

9. Furnari M, Savarino E, Bruzzone L, et al. Reassessment of the role of methane production between irritable bowel syndrome and functional constipation. J Gastrointestin Liver Dis 2012;21:157-163.

10. Arhan P, Devroede G, Jehannin B, et al. Segmental colonic transit time. Dis Colon Rectum 1981;24:625-629.

11. Metcalf AM, Phillips SF, Zinsmeister AR, MacCarty RL, Beart RW, Wolff BG. Simplified assessment of segmental colonic transit. Gastroenterology 1987;92:40-47.

12. Koide A, Yamaguchi T, Odaka T, et al. Quantitative analysis of bowel gas using plain abdominal radiograph in patients with irritable bowel syndrome. Am J Gastroenterol 2000;95:1735-1741.

13. Levitt MD, Ingelfinger FJ. Hydrogen and methane production in man. Ann N Y Acad Sci 1968;150:75-81.

14. Jahng J, Jung IS, Choi EJ, Conklin JL, Park H. The effects of methane and hydrogen gases produced by enteric bacteria on ileal motility and colonic transit time. Neurogastroenterol Motil 2012;24: 185-190, e92.

15. Lee KM, Paik CN, Chung WC, Yang JM, Choi MG. Breath methane positivity is more common and higher in patients with objectively proven delayed transit constipation. Eur J Gastroenterol Hepatol 2013;25:726-732.

16. Pimentel M, Kong Y, Park S. IBS subjects with methane on lactulose breath test have lower postprandial serotonin levels than subjects with hydrogen. Dig Dis Sci 2004;49:84-87.

17. Cloarec D, Bornet F, Gouilloud S, Barry JL, Salim B, Galmiche JP. Breath hydrogen response to lactulose in healthy subjects: relationship to methane producing status. Gut 1990;31:300-304.

18. Morken MH, Berstad AE, Nysaeter G, Berstad A. Intestinal gas in 
plain abdominal radiographs does not correlate with symptoms after lactulose challenge. Eur J Gastroenterol Hepatol 2007;19:589-593.

19. McKay LF, Eastwood MA, Brydon WG. Methane excretion in man--a study of breath, flatus, and faeces. Gut 1985;26:69-74.

20. Miller TL, Wolin MJ. Enumeration of Methanobrevibacter smithii in human feces. Arch Microbiol 1982;131:14-18.

21. Rao SS, Kuo B, McCallum RW, et al. Investigation of colonic and whole-gut transit with wireless motility capsule and radiopaque markers in constipation. Clin Gastroenterol Hepatol 2009;7:537-544.

22. Chan YK, Kwan AC, Yuen H, et al. Normal colon transit time in healthy Chinese adults in Hong Kong. J Gastroenterol Hepatol 2004;19:1270-1275

23. Cho KO, Jo YJ, Song BK, Oh JW, Kim YS. Colon transit time according to physical activity and characteristics in South Korean adults. World J Gastroenterol 2013;19:550-555.

24. Seike K, Koda K, Oda K, Kondo E, Ishizuka M, Miyazaki M.
Relevance of abdominal gas analysis and transit study after colorectal cancer surgery. Scand J Gastroenterol 2003;38:387-391.

25. Ansari R, Sohrabi S, Ghanaie O, et al. Comparison of colonic transit time between patients with constipation-predominant irritable bowel syndrome and functional constipation. Indian J Gastroenterol 2010; 29:66-68.

26. Mollen RM, Claassen AT, Kuijpers JH. [Measurement of colon transit time useful in the evaluation of functional constipation.] Ned Tijdschr Geneeskd 1998;142:357-361. [Dutch]

27. Tornblom H, Van Oudenhove L, Sadik R, Abrahamsson H, Tack J, Simren M. Colonic transit time and IBS symptoms: what's the link? Am J Gastroenterol 2012;107:754-760.

28. Evans JM, Fleming KC, Talley NJ, Schleck CD, Zinsmeister AR, Melton LJ 3rd. Relation of colonic transit to functional bowel disease in older people: a population-based study. J Am Geriatr Soc 1998; 46:83-87. 